\title{
Study on Factors influencing college students to become self-employed Based on ISM and AHP
}

\author{
Leilei Zhao \\ School of Energy Power And Mechanical Engineering, North China Electric Power University, Hebei \\ 071003, China;
}

2773754282@qq.com

Keywords: college students to become self-employed; interpretation structure model (ISM); analytic hierarchy process (AHP); adjacency matrix

\begin{abstract}
In order to futher explore the factors influencing college students to become self-employed, a system of college students to become self-employed influencing factor indexes was made from the perspectives of Society, universities and individuals. ISM idea was adopted for analyzing the links between influencing factors. A 3-layer hierarchal ISM was built for the links. Using AHP, influence forces of the factors were sequenced and an integrated weight system was built. The results show that the hierarchal ISM can effectively build entrepreneurial structure Factors Affecting College Students and directly reflect the hierarchical relationships between the various factors; AHP method to determine the security policy and entrepreneurship, diversification of financing channels, to carry out entrepreneurship contest is the main factors influencing entrepreneurship students. This article provides a reference in order to encourage business students.
\end{abstract}

\section{Introduction}

All levels of society in the face of college students to become self-employed very seriously, Especially after the summer in September 2014 Davos Premier Li issued a call to "popular entrepreneurship and innovation", and college students start to become the focal point. But subsequent entrepreneurship among university students can be seen very few success stories, Therefore, how to stimulate their interest and develop their ability to improve their success rate has become the theorists and practitioners focus of attention. From the existing literature, our scholars of entrepreneurship students are very much, but basically focused on the theoretical study of business students, with ISM and AHP in very few specific studies. Therefore, I will use the ISM and AHP factor analysis of the impact by business students, to determine the mutual relationships between the different levels of the same level and influencing factors. Using AHP to determine the influence of each factor in the model ranking. To provide a scientific basis for further encourage business students.

\section{ISM to the factors influencing college students to become self-employed}

\subsection{Influence Factors}

Table 1 Influence Factors

\begin{tabular}{llc}
\hline NO. & Factors & Symbol \\
\hline 1 & Entrepreneurial environment & $S_{2}$ \\
2 & Quality of individual entrepreneurs & $S_{3}$ \\
3 & Social entrepreneurial environment & $S_{4}$ \\
4 & Entrepreneurship education in universities & $S_{5}$ \\
5 & Personal entrepreneurial awareness and ability & $S_{6}$ \\
6 & Guarantee business and policy & $S_{7}$ \\
7 & Diversification of financing channels & $S_{8}$ \\
8 & Pay attention to intellectual property protection & $S_{9}$ \\
9 & Recruitment of professional business mentor & $S_{10}$ \\
\hline
\end{tabular}




\begin{tabular}{lll}
\hline 10 & Offer entrepreneurship education courses & $S_{11}$ \\
11 & Carry on entrepreneurship contest & $S_{12}$ \\
12 & Participate in entrepreneurship practice & $S_{13}$ \\
13 & $\begin{array}{l}\text { Opportunities to participate in development } \\
\text { activities }\end{array}$ & $S_{14}$ \\
\hline
\end{tabular}

\subsection{Determining binary relation, building reachability matrix and Reduced matrix}

After pairwise comparisons, visually determine the binary relationship between the factors, and within two junction box factors are identified by the symbol V, A and X.

Where $\mathrm{V}$ represents the rows in the table factors directly affect the factors of the column, $\mathrm{A}$ column in the table row factors has a direct impact on the factors, $\mathrm{X}$ represents the mutual influence. According transitive binary relation between factors inferred binary relations between elements, and is represented by the bracketed identifiers. Creating a chart.

\begin{tabular}{|c|c|c|c|c|c|c|c|c|c|c|c|c|c|}
\hline (A) & (A) & (A) & (A) & (A) & (A) & (A) & (A) & (A) & (A) & (A) & A & $\mathrm{A}$ & $S_{1}$ \\
\hline & & (A) & (A) & (A) & (A) & (A) & (A) & & $\mathrm{A}$ & A & & $S_{2}$ & \\
\hline (A) & (A) & (A) & (A) & (A) & & & & A & A & & $S_{3}$ & & \\
\hline & & & & & A & A & A & & & $S_{4}$ & & & \\
\hline & & $\mathrm{A}$ & A & A & & & & & $S_{5}$ & & & & \\
\hline A & A & $\mathrm{A}$ & $\mathrm{A}$ & & & & & $S_{6}$ & & & & & \\
\hline & & & & & & & $S_{7}$ & & & & & & \\
\hline & & & & & & $S_{8}$ & & & & & & & \\
\hline & & & & & $S_{9}$ & & & & & & & & \\
\hline & & & & $S_{10}$ & & & & & & & & & \\
\hline & & & $S_{11}$ & & & & & & & & & & \\
\hline & & $S_{12}$ & & & & & & & & & & & \\
\hline & $S_{13}$ & & & & & & & & & & & & \\
\hline$S_{14}$ & & & & & & & & & & & & & \\
\hline
\end{tabular}

Fig. 1 Effect diagram

\subsection{Extracting skeleton matrix}

$\begin{array}{ccccccccccccccc}\cdots & S_{1} & S_{2} & S_{3} & S_{4} & S_{6} & S_{5} & S_{7} & S_{8} & S_{9} & S_{13} & S_{14} & S_{10} & S_{11} & S_{12} \\ S_{1} & 0 & 0 & 0 & 0 & 0 & 0 & 0 & 0 & 0 & 0 & 0 & 0 & 0 & 0 \\ S_{2} & 1 & 0 & 0 & 0 & 0 & 0 & 0 & 0 & 0 & 0 & 0 & 0 & 0 & 0 \\ S_{3} & 1 & 0 & 0 & 0 & 0 & 0 & 0 & 0 & 0 & 0 & 0 & 0 & 0 & 0 \\ S_{4} & 0 & 1 & 0 & 0 & 0 & 0 & 0 & 0 & 0 & 0 & 0 & 0 & 0 & 0 \\ S_{6} & 0 & 0 & 1 & 0 & 0 & 0 & 0 & 0 & 0 & 0 & 0 & 0 & 0 & 0 \\ S_{5} & 0 & 1 & 1 & 0 & 0 & 0 & 0 & 0 & 0 & 0 & 0 & 0 & 0 & 0 \\ S_{7} & 0 & 0 & 0 & 1 & 0 & 0 & 0 & 0 & 0 & 0 & 0 & 0 & 0 & 0 \\ S_{8} & 0 & 0 & 0 & 1 & 0 & 0 & 0 & 0 & 0 & 0 & 0 & 0 & 0 & 0 \\ S_{9} & 0 & 0 & 0 & 1 & 0 & 0 & 0 & 0 & 0 & 0 & 0 & 0 & 0 & 0 \\ S_{13} & 0 & 0 & 0 & 0 & 1 & 0 & 0 & 0 & 0 & 0 & 0 & 0 & 0 & 0 \\ S_{14} & 0 & 0 & 0 & 0 & 1 & 0 & 0 & 0 & 0 & 0 & 0 & 0 & 0 & 0 \\ S_{10} & 0 & 0 & 0 & 0 & 1 & 0 & 0 & 0 & 0 & 0 & 0 & 0 & 0 & 0 \\ S_{11} & 0 & 0 & 0 & 0 & 1 & 1 & 0 & 0 & 0 & 0 & 0 & 0 & 0 & 0 \\ S_{12} & 0 & 0 & 0 & 0 & 1 & 1 & 0 & 0 & 0 & 0 & 0 & 0 & 0 & 0\end{array}$




\subsection{Drawing interpretation structure model}

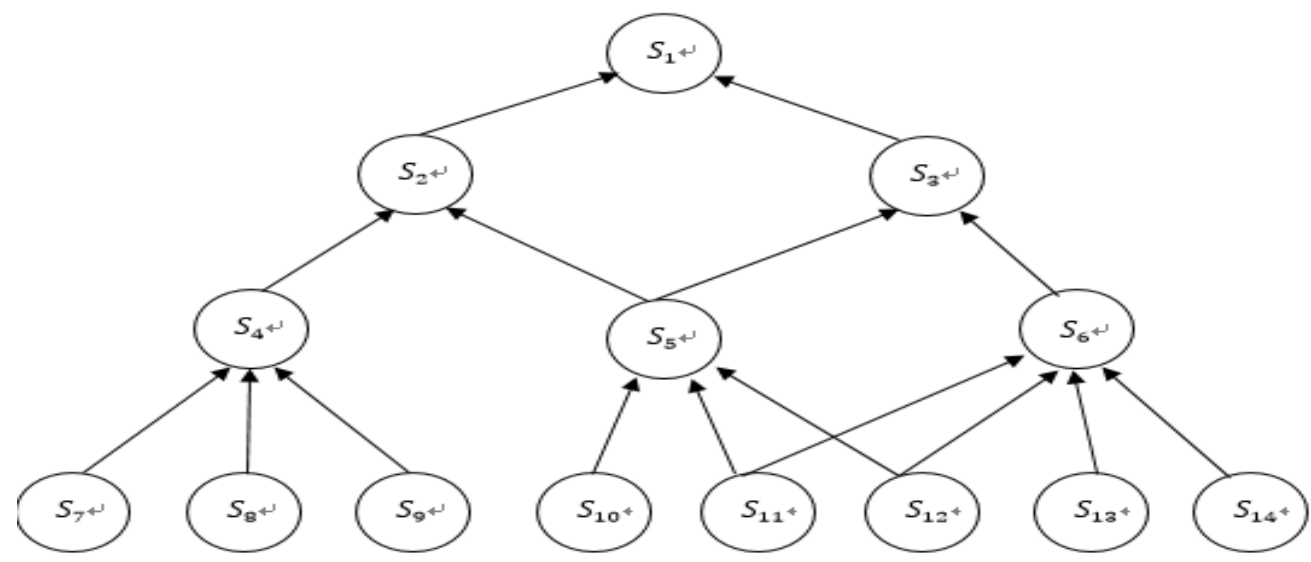

Fig. 2 Drawing interpretation structure model

\section{Analysis of influence factors by AHP}

\subsection{Construct pairwise comparison judgment matrix and its processing}

factors corresponding levels to determine the pairwise comparison matrix element values . Judgment matrix element values reflect the people's awareness of the relative importance of certain factors, the general 1-9 or its inverse scaling method.

Calculated using the root mean square:

\subsection{Consistency check}

$$
\begin{gathered}
V_{i}=\sqrt[n]{I_{1} I_{2} I_{3} \ldots I_{n}} \\
W_{i}=\frac{V_{i}}{\sum_{1}^{n} V_{i}}
\end{gathered}
$$

$$
\text { C.I. }=\frac{\lambda_{\max }}{n-1}
$$

$$
\lambda_{\max }=\frac{1}{n} \sum_{1}^{n} \frac{(A W)_{i}}{W_{i}}
$$

C.R. $=\frac{\text { C.I. }}{R . L .}<0.1$ qualified

Table 2 The results of the consistency check

\begin{tabular}{ccl}
\hline factor & C.R. & result \\
\hline$S_{1}$ & 0 & qualified \\
\hline$S_{2}$ & 0 & qualified \\
\hline$S_{3}$ & 0 & qualified \\
\hline$S_{4}$ & 0.0312 & qualified \\
\hline$S_{5}$ & 0 & qualified \\
\hline$S_{6}$ & 0.0155 & qualified \\
\hline
\end{tabular}

Table 3 Correlation matrix

\begin{tabular}{lllll}
\hline & \multicolumn{1}{c}{$S_{4}$} & \multicolumn{1}{c}{$S_{5}$} & \multicolumn{1}{c}{$S_{6}$} & $V_{i}$ \\
\cline { 2 - 4 } & 0.5834 & 0.1250 & 0.2916 & \\
\hline$S_{7}$ & 0.5821 & 0 & 0 & 0.3396 \\
\hline$S_{8}$ & 0.3484 & 0 & 0 & 0.2032 \\
\hline$S_{9}$ & 0.0695 & 0 & 0 & 0.0405 \\
\hline$S_{10}$ & 0 & 0.2857 & 0 & 0.0357 \\
\hline$S_{11}$ & 0 & 0.1429 & 0.0592 & 0.0351 \\
\hline$S_{12}$ & 0 & 0.5714 & 0.2422 & 0.1421 \\
\hline$S_{13}$ & 0 & 0 & 0.3940 & 0.1149 \\
\hline$S_{14}$ & 0 & 0 & 0.3046 & 0.0888 \\
\hline
\end{tabular}


8 program from good to bad sort of $S_{7}, S_{8}, S_{12} 、 S_{13} 、 S_{14}, S_{9}, S_{10}$.

\section{Summary}

Finally found security, financing and business diversification policy, carry out entrepreneurial class competition are the main factors that affect college students start their own businesses, other factors are auxiliary. So, at first, in terms of security policy and entrepreneurship, the Government should introduce some workable and effective policies. Secondly, in the promotion of diversified financing channels, countries should establish a special fund to achieve student college students venture diversified financing channels. Then, in undertaking entrepreneurial competition, universities should actively cooperate with the government's call to carry out some regular classes start the game, invite some business owners and experts as evaluation of student entrepreneurship program evaluation guide. Finally, college students should actively participate in entrepreneurial practice, from start to understand what is, from a practical exercise their own activities, and accumulated experience for the future of their own businesses.

\section{References}

[1]LI Nai-wen, XU Meng-Hong, NIU Li-xia, et al. Study on Factors Influencing Miners' Habitual Violation Behavior Based on ISM and AHP.China Safety Science Journal.Vol.22 No.8 Aug.2012

[2]YANG Jia-Yong: Research On Promoting Factors And Entrepreneurial Effect----In A University As An Example (Doctor, YUNNAN University, China, 2013).p.30 